\title{
Optimisation of perishable inventory items with geometric return rate of used product
}

\author{
$\underline{\text { S. Sanni }}^{\text {a }}$, B. O’Neill ${ }^{\text {a }}$, Z. Jovanoski ${ }^{\text {a }}$ and H.S. Sidhu ${ }^{\text {a }}$ \\ ${ }^{a}$ School of Physical, Environmental and Mathematical Sciences, UNSW Canberra. \\ Email: Smaila.sanni@student.adfa.edu.au
}

\begin{abstract}
We present an inventory model for a firm facing a price-sensitive demand rate and in which the deterioration rate of item is constant. For this constant rate of perishable inventory model, we incorporate a return rate of used items which accounts for the impact of reused products on inventory decisions. We broaden the model scope by allowing two cases of purchase payments: payment at the time of delivery and prepayment before delivery. The model is developed and analysed under a cost function which is linear with respect to the firm's order size and the total-item holding cost. The firm seeks to maximise profit-rate by appropriate choice of the price, return rate and cycle length. We present the conditions that guarantee the existence and uniqueness of the optimal solutions to the inventory problem. To investigate the application of the proposed model we conduct a brief numerical study and set up a sensitivity analysis table to assess the effect of changes in the model parameters on the firm's optimal policy. The design serves two purposes. Firstly, it provides an objective way of quantifying how the optimal policy response to changes in the model parameters and, secondly, it helps to identify the set of changes in the input that yields a new optimal.
\end{abstract}

Keywords: Optimal policy, order size, return rate, re-use 
Sanni et al., Optimization of perishable inventory items with geometric return rate of used products

\section{INTRODUCTION}

Harris (1913) introduced the square root-formula of the economic order quantity (EOQ) model for calculating the best order quantity a firm should hold so as to minimise the total cost of inventory. Many authors have considered several modifications and extensions to the basic EOQ incorporating more realistic factors into the model (see, for example, Philip (1974), Deb and Chaudhuri (1987), Detta and Pal (1990), Chang (2010) and Sanni and Chigbu (2017)). Most of these extant models ignored the impact of return products via reverse logistics program on the inventory decision. Hawks (2006) defined reverse logistics as the process of moving goods from the point of destination to the point of origin for the purpose of recapturing value or proper disposal. The reverse flow of goods affects inventory level and total inventory cost, and it should be taken into account when analysing inventory systems. Gains due to reverse logistics include enhanced customer satisfaction, decreased inventory levels and reduced cost.

The core components of reverse logistics are gatekeeping, collection, sortation, and disposition (Horvath et al (2005) and Hawks (2010) which can be collectively referred to as central return centre. The expenses associated with the operation of reverse logistic have direct effect on inventory decision. In the recent past, reverse logistics has attracted a great deal of attention from researchers in inventory modelling.

While reverse logistics configuration is new and critical to inventory modelling, demand rate and deterioration have long been recognised as essential aspects of inventory model. Bose et al (1998) proposed a finite horizon EOQ model for items with linear demand rate and constant deterioration. Taleizadeh $(2014 \mathrm{a}, 2014 \mathrm{~b})$ introduced a number of EOQ models with constant decay rate and prepayment options. Chen et al (2016) developed an EOQ model for inventory system in which return of used product is allowed and in which items in the system do not deteriorate.

In the literature, we have seen that the focus is primarily on methods for determining appropriate order size for minimising system cost. In these models, little or no attention is given to the reverse flow of items into the inventory. Hence, the problem of including reverse logistic arrangement in inventory decisions remains largely as open research area.

In this paper, we study the EOQ model with reverse logistics program in a specific form allowing for linear demand rate and constant rate of deterioration of items. We formulate an optimisation problem for the firm and obtain optimal inventory policy. The results are demonstrated using numerical studies.

\section{MATHEMATICAL MODEL AND ANALYSIS}

We consider the problem of profit maximisation for a firm with reverse logistics scheme. The demand is affected by the unit selling price the firm sets for its goods and a constant fraction of items that deteriorate over the cycle time. Suppose that the firm wishes to maximise its profits for an appropriate choice of the order size $y$, the unit selling price $p$ and the return rate parameter $\delta$. For $\delta>0, T>0$ and $p>0$, we have $y>0$ so that the profit consists of revenue from sales of new and returned items and is reduced by purchase cost, holding cost and the cost of running the reverse logistic system. Therefore, for all $T>0$ the average profit-rate is given by

$$
\pi(p, \delta, T)=\left[p+\left(b_{1}-b_{2}\right) R(\delta)\right] r(p)-\frac{1}{T}\left[a+b_{0} y(p, T)+c_{0} h(p, T)+c_{1}(\delta)\right]
$$

where $b_{0}$ is the purchase cost per unit of item, $a$ is the fixed cost per order, and $c_{0}$ is the carrying cost of item per unit per unit time. The parameter $b_{1}$ represents the unit cost of collecting, holding, sorting and handling a returned item, which includes the collection fees paid by the firm to consumers. The parameter $b_{2}$ is the unit price of a used returned product sold by the firm to its supplier, here we assume a positive salvage value $b_{1}-$ $b_{2}$ of collected used product. For similar treatment (see Chen eta l (2016)).

Some expenses are associated with running a reverse logistic scheme. The investment cost of the firm operating a reverse logistic program is assumed to be a quadratic function of the return rate coefficient, $c_{1}(\delta)=c \delta^{2}$. This assumption ensures that the incremental rate of the operational cost of the reverse logistic program depends on the return rate. Following Chen et al (2016) it is assumed that products sold in the past replenishment cycles can be collected in the current replenishment cycle and that the return rate for product sold in the past $i$-th replenishment cycle follows a geometric series with initial value as the return rate coefficient $\delta$ and common ratio $\mu, 0 \leq \mu \leq 1$. The return rate for the products sold in the past $i$-th replenishment cycle is thus given by $\delta \mu^{i-1}$ and the sum of the returned rate for used products in the current replenishment cycle is represented by $R(\delta)=\sum_{i=1}^{\infty} \delta \mu^{i-1}=\delta /(1-\mu)$. It is only reasonable to assume the total number of collected used items be non-negative and that this number should not excess the number of 
product sold in each replenishment cycle. Thus, the scope of the firm's profit maximisation is limited by the constraint $0<R(\delta) \leq 1$.

To determine the inventory level and total-item hold time, let the inventory starts at the order size $y$ which then depletes due to the simultaneous effects of demand and deterioration until it becomes empty at the cycle time $t=T$. At this time it is replenished by a new order and the process repeats. The equation governing the inventory process $I(p, T)=\{I(t \mid p, T) \mid 0 \leq t \leq T\}$ is

$$
I(0 \mid p, T)=y(p, T) \quad \frac{d I}{d t}(t \mid p, T)+\theta I(t \mid p, T)=-r(p) \text { for all } 0 \leq t \leq T,
$$

where $\theta$ is the constant proportion of deterioration of items per cycle time. The solution to the inventory equation is:

$$
I(t \mid p, T)=r(p) \frac{\exp (\theta(T-t))-1}{\theta} \text { for } 0 \leq t \leq T
$$

The order size and the total item-holding time are given respectively by

$$
y(p, T)=r(p) \frac{\exp (\theta T)-1}{\theta}, \quad h(p, T)=\int_{0}^{T} I(t \mid p, T) d t=r(p) \frac{\exp (\theta T)-\theta T-1}{\theta^{2}} .
$$

Putting Eqn (3) and Eqn (4) into Eqn (1), we can write the profit function for the firm as

$$
\pi(p, \delta, T)=\left(p+\frac{\left(b_{1}-b_{2}\right) \delta}{\mu}+\frac{c_{0}}{\theta}-\frac{1}{\theta T}\left(b_{0}+\frac{c_{0}}{\theta}\right)(\exp (\theta T)-1)\right) r(p)-\frac{a+c \delta^{2}}{T}
$$

There is no explicit solution for the decision variables using the profit function in Eqn (5) because of the presence of $1 /(\theta T)$ and $\exp (\theta T)$ in the function which do not augur well for solving explicitly for $T$. The exact solution for the cycle length $T$ can be described in terms of the Lambert $W$-function, also called the Product Log function. Although it is possible to obtain the exact solution using iterative schemes, we adopt quadratic approximation of the exponential term for the sake of analytic and computational convenience. Some authors have favoured this approach (see, e.g., Taleizadeh (2014a)). For specific structure of the rate of withdrawal of items from the inventory system, let the firm possess a linear demand rate $r(p)=m-k p$ where $m$ is the fixed demand and $k$ is the elasticity coefficient, the average profit-rate for this model is

$$
\pi(p, \delta, T)=\left(p+\frac{\left(b_{1}-b_{2}\right) \delta}{1-\mu}+\frac{c_{0}}{\theta}-\left(b_{0}+\frac{c_{0}}{\theta}\right)\left(1+\frac{1}{2} \theta T\right)\right)(m-k p)-\frac{a+c \delta^{2}}{T} .
$$

Therefore, the optimisation problem the firm is facing is $\max _{P, \delta, T>0}\{\pi(p, \delta, T)\}$ subject to $0<R(\delta) \leq 1$.

The firm seeks to maximise the average profit rate by appropriate choice of the price $(p)$, return rate $(\delta)$ and cycle length $(T)$, which by Eqn (4) determines the order size. We solve the optimisation problem by considering four possible scenarios. Firstly, we consider the price level and the return rate to be fixed exogenous values and we determine the optimal cycle length for a given price and return rate. Secondly, we determine the optimal price level for a fixed cycle length and return rate. Thirdly, we consider the price level to be a fixed exogenous value and we will determine the optimal cycle length and optimal return rate. Finally we will consider the case where the price level, the return rate and the cycle length are all decision variables limited only by the return rate constraint. The following propositions give the solutions to all the four cases mentioned above (all proofs in appendix).

Proposition 1. For any given price level $p$ and fixed return rate $\delta$, the optimal cycle length for the firm is given by

$$
\widehat{T}=\sqrt{\frac{2\left(a+c \delta^{2}\right)}{c_{0}+b_{0} \theta} \cdot \frac{1}{m-k p}} .
$$

Proposition 2. For any fixed cycle length and return rate, an endogenous price level is given by

$$
\hat{p}=\frac{1}{2 k}\left[m-k\left(\frac{\left(b_{1}-b_{2}\right) \delta}{1-\mu}+\frac{c_{0}}{\theta}-\left(b_{0}+\frac{c_{0}}{\theta}\right)\left(1+\frac{1}{2} \theta T\right)\right)\right] .
$$


Proposition 3. For a fixed exogenous price level, the optimal cycle length and the optimum return rate are respectively given by

$$
\begin{gathered}
\hat{T}=\sqrt{\frac{2 a}{(m-k p)\left[\left(c_{0}+b_{0} \theta\right)-\psi\right]}} \\
\hat{\delta}=\frac{\left(b_{1}-b_{2}\right)(m-k p)}{2 c(1-\mu)} \hat{T} \leq 1-\mu,
\end{gathered}
$$

where $\psi=\frac{\left(b_{1}-b_{2}\right)^{2}(m-k p)}{2 c(1-\mu)^{2}}$.

Remark: If the optimal return rate $\hat{\delta}$ falls outside the interval $(0,1-\mu]$, violating the scope of optimisation, it is set as $\hat{\delta}=1-\mu$ and then proceed to obtain a new optimal cycle length.

Proposition 4. If the price level, the return rate and the cycle length are decision variables and are endogenously determined by the firm, then the optimal solutions can be obtained using Karush-Kuhn-Tuckers' (KKT) conditions as follows;

We construct the Lagrangian:

$$
\mathcal{L}(p, \delta, T, \lambda)=\pi(p, \delta, T)-\lambda\left(1-\frac{\delta}{1-\mu}\right) .
$$

Then the KKT necessary conditions for a point be maximum are obtained (see appendix).

The conditions for payment of items and their impact on the unit purchase cost can affect the firm's inventory policy. Prepayment is an essential option of procurement. Similar to Taleizedah (2014a), we define the prepayment as $b_{0}^{*}=b_{0}\left[1+\frac{\alpha v i}{n}\left(\frac{n+1}{2}\right)\right]$, where $n$ is the fixed number of prepayments at equal interval of $v$ times apart, $\alpha$ is the proportion of purchase cost to be paid as multiple advanced payment and $i$ is the capital cost rate per unit time. With prepayment, the unit $\operatorname{cost} b_{0}$ in the profit function is replaced with the prepayment formula $b_{0}^{*}$.

\section{NUMERICAL STUDY}

Example 1. An inventory problem with prepayment option, Logistic program and constant decay is studied. We consider a distributor of certain electronic product is reviewing its inventory policy for one particular model. The distributor's supplier requires it to prepay $40 \%$ of the purchase cost by making a series of 3 fixed prepayments at equal interval of 0.2 year. The demand for the product is 800 unit per month with elasticity coefficient of 0.65. The other relevant inventory data are: $a=\$ 60$ per order, $b_{0}=\$ 12$ per unit, $b_{1}=\$ 3.5$ per unit, $b_{2}=\$ 1$ per unit, $c_{0}=\$ 0.05$ per unit per year, $c=125, \mu=0.15, \theta=0.1$. $i=\$ 0.35$ per year.

Using the solution in Eqn (10) we obtain the optimal return rate $\hat{\delta}=0.8$, and the optimal price $\hat{p}=\$ 620.56$, and other outcomes are:

$$
\hat{y}=299.082 \text { units } \quad \widehat{T}=0.7270 \quad \widehat{\pi}=\$ 241,840.67 .
$$

Figure 1 shows the firm's profit function for various prices and return rates. 


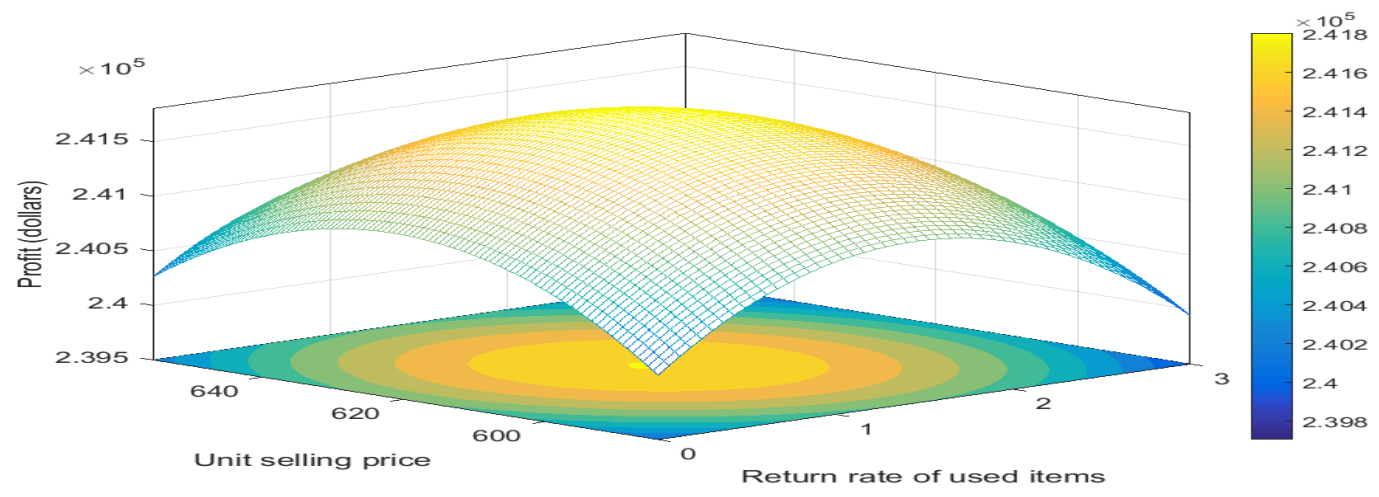

Figure 1. Profit function with respect to price and return rate. The concavity of the profit function with respect price and return rate indicates that unique global optimal policy exist.

Example 2. An Inventory problem with logistic program, constant decay and no prepayment option is studied. Consider the inventory data from Example 3 of Chen et al (2016): $b_{0}=10, b_{1}=3, b_{2}=1, c_{1}=10000$, $a=3000, c_{0}=0.8, \mu=0.2$ and the demand function is $r(p)=1000-15 p$. In addition let a constant fraction of the on-hand inventory deteriorate per unit time $\theta=0.3$.

Using the solution in Eqn (8) and Eqn (9) we obtain the optimal return rate $\hat{\delta}=0.0843$, and the optimal price $\hat{p}=\$ 40.15$, and other outputs are:

$$
\hat{y}=879.09 \text { units } \hat{T}=1.6955 \quad \widehat{\pi}=8735.41 .
$$

In comparison, it is observed that the model proposed in Chen et al (2016) which ignores the impact of deterioration of items overestimates the optimal return and the optimal order size, and underestimate the optimum unit selling price.

In order to gain more insight of the above model we perform sensitivity analysis on some input parameters by generating a table that shows what the optimal solutions would be if the true inputs were each as given in Table 1.

From Table 1 when the deterioration rate $\theta=$ 0.00001 , the solutions are closely similar to the results in Chen et al (2016) where the impact of deterioration was completely ignored. Further, it can be seen

Table 1. Sensitivity analysis on the deterioration rate and the investment scaling parameter

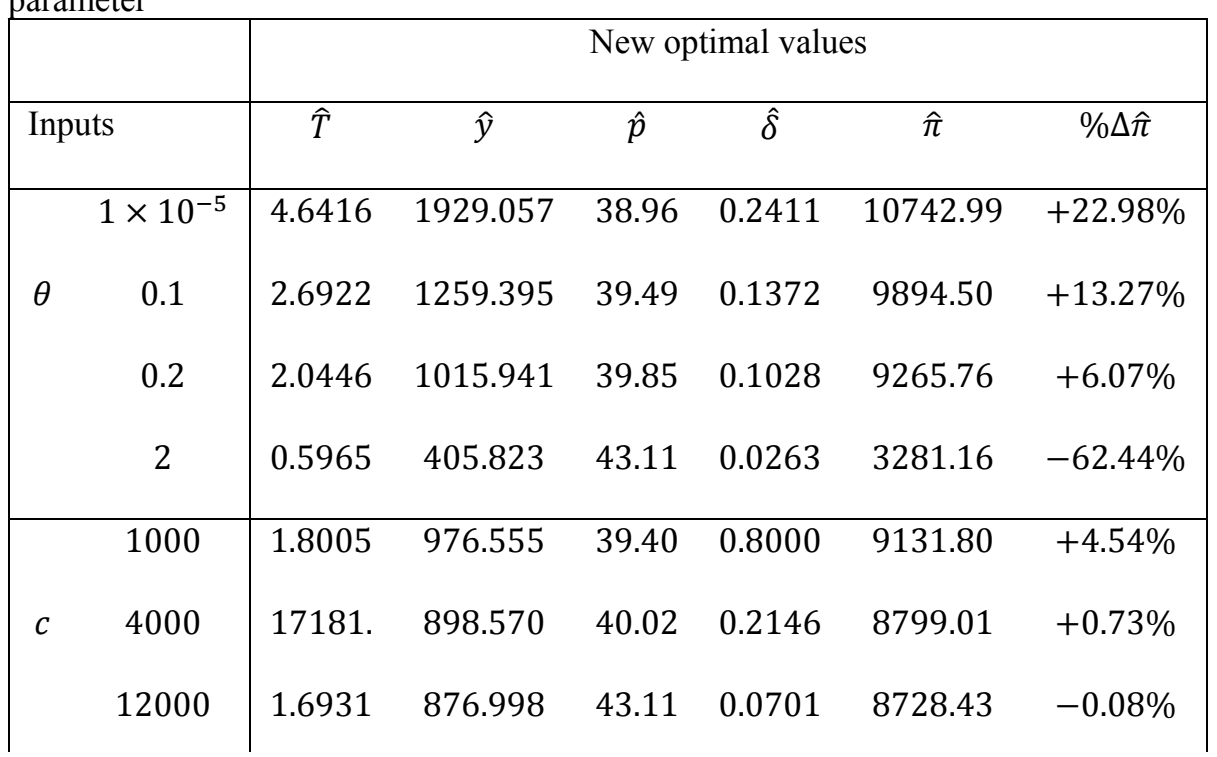

that the optimal profit

rate decreases as the deterioration rate increases. An increase in the reverse logistics investment scaling parameter leads to a decrease in profit. 


\section{CONCLUSION}

We introduced an economic order quantity (EOQ) model with reverse logistics strategy for deteriorating products. We examined the impacts of deterioration of items and reverse logistics program on inventory management decision. Our work complements the EOQ literature by showing that prepayments options, investment in reverse logistics program and deterioration of items affect the firm's optimal choices.

We confirm the analytical results using numerical studies. Sensitivity analysis table is presented to show how the firm's optimal policy respond to changes in model input values. One could further the model by considering situation where items have expiry date.

\section{REFERENCES}

Bose, S., Goswami, A., and Chaudhuri, K.S. (1995). An EOQ model for deteriorating items with linear timedependent demand rate and shortages under inflation and time discounting. Journal of Operational Research Society, 46(6), 771-782.

Chang, H.J. (2010). A partial backlogging inventory model for non-instantaneous items with stock-dependent consumption rate under inflation. Yugoslav Journal of Operations Research, 20(1), 35-54.

Chen, C.-K., Weng, T.-C., and Lo, C.-C. (2016). Optimal replenishment quantity for new products and return rate of used products for a retailer. Applied mathematical Modelling, 40, 9754-9766.

Deb, M., and Chaudhuri, K. (1987). A note on the heuristic for replenishment of trended inventories considering shortages. Journal of Operational Research Society, 38 (5), 459-463.

Detta, T.K., and Pal, A.K. (1990). A note on inventory model with inventory level dependent demand rate. Journal of Operational Research Society, 41, 971-975

HARRIS, F.W. (1913) How many parts to make at once. Factory: The Magazine of Management, 10(2), 135$136,152$.

Hawks, K. (2006). What is reverse logistics? Reverse Logistics Magazine, Winter/Spring edition.

Horvath, P.A., Autry, C.W., and Wilcox, W.E. (2005). Liquidity implications of reverse logistics for retailers: A markov chain approach. Journal of Retailing, 81(2), 191-203.

Philip, G.C. (1974). A generalized EOQ model for items with Weibull distribution deterioration. AIIE Transactions, 6, 159-162.

Sanni,S.S. and O’Neill, B. (2016). Profit optimisation for deterministic inventory system with linear cost. Under review.

Sanni, S.S., and Chigbu, P.E. (2017). Optimal replenishment policy for items with three-parameter Weibull deterioration, stock-level dependent demand and partial backlogging. Yugoslav Journal of Operations Research, DOI: 10.2298/YJOR151120019S

Taleizadeh, A.A. (2014a). An economic order quantity model for deteriorating items in a purchasing system with multiple prepayments. Applied Mathematical Modelling, 38 (23), 5357-5366.

Taleizadeh, A.A. (2014b). An EOQ model for partial and advance-payments for evaporating items. International Journal of Production Economics, 155, 185-193. 
Sanni et al., Optimization of perishable inventory items with geometric return rate of used products

\section{APPENDIX}

In this appendix, we set the KKT conditions for the firm's constrain maximisation problem.

Consider Equation (10) and taking partial derivative with respect to the relevant decision variable, we have the first-order necessary conditions (KKT) for the nonlinear optimisation:

$$
\begin{array}{llcc}
\frac{\partial \mathcal{L}}{\partial T}=-\frac{1}{2}\left(c_{0}+b \theta\right)(m-k p)+\frac{a+c_{1} \delta^{2}}{T^{2}} \leq 0 & T \geq 0 & T\left(-\frac{1}{2}\left(c_{0}+b \theta\right)(m-k p)+\frac{a+c \delta^{2}}{T^{2}}\right)=0 \\
\frac{\partial \mathcal{L}}{\partial p}=m-2 k\left(p+\frac{1}{2} \Omega\right) \leq 0 & p \geq 0 & p\left(m-2 k\left(p+\frac{1}{2} \Omega\right)\right)=0 \\
\frac{\partial \mathcal{L}}{\partial \delta}=\frac{\left(b_{1}-b_{2}\right)(m-k p)}{1-\mu}-\frac{2 c_{1} \delta}{T}-\frac{\lambda}{1-\mu} \leq 0 & \delta \geq 0 & \delta\left(\frac{\left(b_{1}-b_{2}\right)(m-k p)}{1-\mu}-\frac{2 c \delta}{T}-\frac{\lambda}{1-\mu}\right)=0 \\
\frac{\partial \mathcal{L}}{\partial \lambda}=\frac{\delta}{1-\mu}-1 \leq 0 & \lambda \geq 0 & \lambda\left(\frac{\delta}{1-\mu}-1\right)=0,
\end{array}
$$

where $\Omega=\left(\frac{\left(b_{1}-b_{2}\right) \delta}{1-\mu}+\frac{c_{0}}{\theta}-\left(b_{0}+\frac{c_{0}}{\theta}\right)\left(1+\frac{1}{2} \theta T\right)\right)$ and $\lambda$ is the Lagrange multiplier. The resulting systems of inequalities and equations can be solved to find the optimum values. Sanni and O'Neill (2017) have showed for a general class of inventory optimisation problems that the profit rate function is quasi-concave. Thus, the KKT necessary conditions are sufficient to guarantee global optimal policy for the firm. 